\title{
Design tool for the actuation control of a microfluidic valve using fluid-structure interaction partitioned approach
}

\author{
A.Aissa Berraies1 ${ }^{1}$, F.Auricchio $^{1}$, F.Butera ${ }^{3}$ and EH. van Brummelen ${ }^{2}$ \\ ${ }^{1}$ Computational Mechanics \& Advanced Materials Group, Department of Civil Engineering and \\ Architecture \\ Universita Degli Studi di Pavia (UniPV) \\ Via Adolfo Ferrata, 3, 27100 Pavia, Italy \\ e-mail: ahmed.aissaberraies01@universitadipavia.it, Ferdinando.auricchio@unipv.it \\ 2 Multiscale Engineering Fluid Dynamics Group, Department of Mechanical Engineering \\ Technische Universiteit Eindhoven (TU/e) \\ 5612 AZ Eindhoven, Netherlands \\ e-mail: e.h.v.brummelen@tue.nl \\ ${ }^{3}$ Dolphin Fluidics Srl \\ Via Leonardo da Vinci, 40 - 20094 Corsico (Milano), Italy \\ e-mail : f.butera@dolphinfluidics.com - Web page: www.dolphinfluidics.com
}

\begin{abstract}
Several concepts have been developed for the actuation of micro-valves to overcome challenges [1] regarding the reduction of flow leakage and power consumption, the improvement of response time and high work density and the ability to operate with both liquid and gas [2].

Pilot-valves belong to the class of passive valves operating in two stages. They are essentially designed to control the flow-rate with enhanced accuracy. However, specific properties required for its design cannot be extracted experimentally. Hence, numerical fluid-structure interaction (FSI) $[3,4,5]$ simulations are used to study the valve operating conditions, to help eventually optimizing the product and overcoming design limitations.

The methods used to solve FSI problems in the literature follow either a monolithic approach or a partitioned iterative approach [6]. While the first approach offers stability and robustness, it is generally not adopted within commercial software due to solvers modularity restrictions. Recent advances have allowed the implementation of coupling solvers to perform FSI simulations using partitioned approach. However, this method might have limitations in terms of stability and robustness, especially when dealing with strongly coupled simulations invoking high non-linearities, particularly for incompressible fluids causing high added-mass effects [7, 8, 9].

This study presents the relevant methodologies and techniques employed to study the micro-scale pilot-valve developed by Dolphin Fluidic company. Mainly, we focus on the numerical approaches to describe the incompressible fluid behavior, and the membrane deformation during valve opening/closing phases. The partitioned iterative method is used to solve the deforming boundaries occurring within the fluid-structural interfaces. The structural domain consists of a thin membrane made of rubber-like incompressible hyperelastic material. The density ratio gives rise to a high added mass effect which leads to numerical instabilities. We illustrate the instabilities encountered and the numerical methods followed to mitigate them and develop a design tool for the microfluidic valve problem.
\end{abstract}

\section{REFERENCES}

[1] Oh, Kwang W Oh, and Chong H Ahn. A review of microvalves, Journal of micromechanics and microengineering $16.5,2006$. 
[2] Natarajan et al, Analysis of Membrane Behavior of a Normally Closed Microvalve Using a Fluid Structure Interaction Model, 2017.

[3] Zienkiewicz, O.C. and Taylor, R.L. The finite element method. 6th Edition, ButterworthHeinemann, 2005.

[4] Farhat, CFD-Based Nonlinear Computational Aeroelasticity, Encyclopedia of Computational Mechanics, Vol. 3: Fluids, E. Stein, R. Borst, and T. Hughes, eds., Wiley, New York, pp. 459-480, 2004.

[5] Qun Zhang Song Cen, Multiphysics Modeling Numerical Methods and Engineering Applications, 2016.

[6] van Brummelen EH, Geuzaine, P., Fundamentals of Fluid-Structure Interaction, Encyclopedia of Aerospace Engineering, 2010.

[7] van Brummelen EH. Partitioned iterative solution methods for fluid-structure interaction, Int. J. Numer. Meth. Fluids, 2010.

[8] Causin P, Gerbeau JF, Nobile F. Added-mass effect in the design of partitioned algorithms for fluid-structure problems, Computer Methods in Applied Mechanics and Engineering; 194:45064527, 2005.
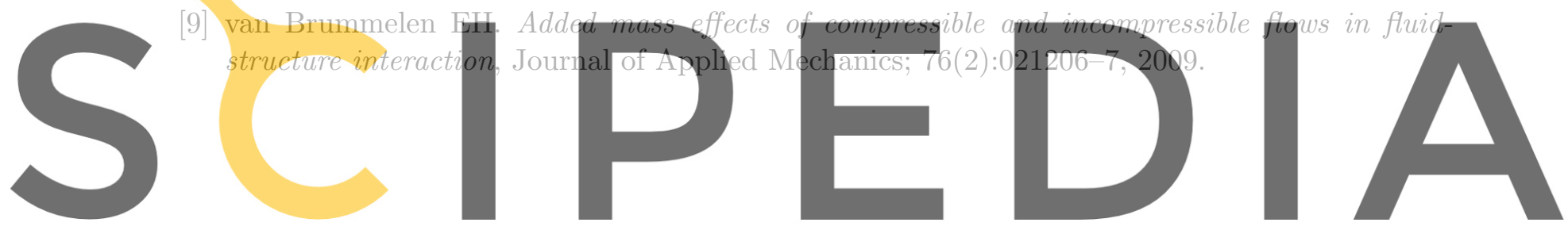